\title{
Modified Block-Pulse Functions Scheme for Solve of Two-Dimensional Stochastic Integral Equations
}

\author{
Mohsen Fallahpour ${ }^{1}$ and Morteza Khodabin ${ }^{1 *}$ \\ ${ }^{1}$ Department of Mathematics, Karaj Branch, Islamic Azad University, Karaj, Iran. \\ *Corresponding author E-mail:m-khodabin@kiau.ac.ir
}

\section{Article Info}

Keywords: Ito integral equation, Modified block-pulse functions, Operational matrix, Two-dimensional integral equation, Volterra-Fredholm integral equation.

2010 AMS: 65C20, 45Axx, 45Bxx, 45Dxx, 60H2O.

Received: 7 May 2019

Accepted: 20 April 2020

Available online: 24 April 2020

\begin{abstract}
In this paper, two-dimensional modified block-pulse functions (2D-MBPFs) method is introduced for approximate solution of 2D-linear stochastic Volterra-Fredholm integral equations so the ordinary and stochastic operrational matrices of integration are utilized to reduce the computation of such equations into some algebraic equations. Convergence analysis of this method is discussed. Finally an illustrative example is given to show the accuracy of the proposed method so the results of it is compared with the block-pulse functions (BPFs) method.
\end{abstract}

\section{Introduction}

Mainly 2D-integral equations furnish the important implement for modeling the engineering and science problems [1, 2]. We have used the variant methods for solving 2D-linear stochastic integral equations in $[3,4,5,6,7]$ that the BPFs method is one of these methods. The BPFs are very common in use, but it seems that their convergence is weak. Here the modified block-pulse functions (MBPFs) method is used for deriving approximation solution of 2D-linear stochastic Volterra-Fredholm integral equation of the second kind

$$
g(x, y)=f(x, y)+\int_{0}^{1} \int_{0}^{1} V_{1}(x, y, s, t) g(s, t) d s d t+\int_{0}^{y} \int_{0}^{x} V_{2}(x, y, s, t) g(s, t) d s d t+\int_{0}^{y} \int_{0}^{x} V_{3}(x, y, s, t) g(s, t) d B(s) d B(t),
$$

where $(x, y) \in\left[0, T_{1}\right) \times\left[0, T_{2}\right)$ and

$$
s \leqslant x<t \leqslant y
$$

In (1.1), $g(x, y)$ is the unknown function and the condition (1.2) is necessary.

We organize the paper as follows:

The properties of 2D-MBPFs are introduced in the next section. In Section 3 we solve (1.1) by finding the ordinary and stochastic operational matrices. We depict the error analysis in Section 4. The certitude of the method is evinced by an example in Section 5. Eventually, we afford the brief conclusion in Section 6.

\section{Two dimentional MBPFs}

An $\left(n_{1}+1\right) \times\left(n_{2}+1\right)$-set of 2D-MBPFs $\omega_{a_{1}, a_{2}}(x, y) \quad\left(a_{1}=0,1, \ldots, n_{1}\right) ;\left(a_{2}=0,1, \ldots, n_{2}\right)$ consists of $\left(n_{1}+1\right) \times\left(n_{2}+1\right)$ functions which are defined over district $D$ by $[8]$

$$
\omega_{a_{1}, a_{2}}(x, y)= \begin{cases}1, & (x, y) \in D_{a_{1}, a_{2}} \\ 0, & \text { otherwise }\end{cases}
$$


where

and

$$
D_{a_{1}, a_{2}}=(x, y): x \in I_{a_{1}, \varepsilon}, y \in I_{a_{2}, \varepsilon}
$$

$$
\begin{gathered}
I_{a_{1}, \varepsilon}=\left\{\begin{array}{cc}
{\left[0, k_{1}-\varepsilon\right),} & a_{1}=0 \\
{\left[a_{1} k_{1}-\varepsilon,\left(a_{1}+1\right) k_{1}-\varepsilon\right),} & a_{1}=1(1)\left(n_{1}-1\right) \\
{[1-\varepsilon, 1)} & a_{1}=n_{1},
\end{array}\right. \\
I_{a_{2}, \varepsilon}=\left\{\begin{array}{cc}
{\left[0, k_{2}-\varepsilon\right),} & a_{2}=0 \\
{\left[a_{2} k_{2}-\varepsilon,\left(a_{2}+1\right) k_{2}-\varepsilon\right),} & a_{2}=1(1)\left(n_{2}-1\right) \\
{[1-\varepsilon, 1)} & a_{2}=n_{1},
\end{array}\right.
\end{gathered}
$$

where $n_{1}$ and $n_{2}$ are arbitary positive integers and we have

$$
k_{1}=\frac{T_{1}}{n_{1}}, k_{2}=\frac{T_{2}}{n_{2}} .
$$

From (2.1), we can represent 2D-MBPFs as

$$
\omega_{a_{1}, a_{2}}(x, y)=\omega_{a_{1}}(x) \omega_{a_{2}}(y),
$$

where $\omega_{a_{1}}$ and $\omega_{a_{2}}$ are the one-dimensional MBPFs. Similar to the one-dimensional case, 2D-MBPFs have the elementary properties that are: disjointness, orthogonality and completeness. Also the set of 2D-MBPFs can be written as a vector $\Omega(x, y)$ of dimension $\varsigma_{1}=\left(n_{1}+1\right)\left(n_{2}+1\right) \times 1$ as

$$
\Omega(x, y)=\left[\omega_{0,0}(x, y), \ldots, \omega_{0, n_{2}}(x, y), \ldots, \omega_{n_{1}, 0}(x, y), \ldots, \omega_{n_{1}, n_{2}}(x, y)\right]^{T},
$$

where $(x, y) \in D$. For every $\varsigma_{1}$-vector $K$ from (2.2) we have

$$
\Omega(x, y) \Omega^{T}(x, y) K=\tilde{K} \Omega(x, y),
$$

where $\tilde{K}=\operatorname{diag}(K)$ is a diagonal matrix of dimension $\varsigma_{2}=\left(n_{1}+1\right)\left(n_{2}+1\right) \times\left(n_{1}+1\right)\left(n_{2}+1\right)$. Moreover, for every $\varsigma_{2}$-matrix $H$ we get

$$
\Omega^{T}(x, y) H \Omega(x, y)=\hat{H}^{T} \Omega(x, y),
$$

where $\hat{H}$ is an $\varsigma_{1}$-vector with elements equal to the diagonal entries of matrix $H$.

\subsection{Two dimensional MBPFs expansions}

A function $f(x, y)$ defined over $L^{2}(D)$ can be expanded by the 2D-MBPFs as [8, 9]

$$
f \simeq f_{\varepsilon}=\sum_{a_{1}=0}^{n_{1}} \sum_{a_{2}=0}^{n_{2}} f_{a_{1}, a_{2}} \omega_{a_{1}, a_{2}}=F^{T} \Omega,
$$

where $F$ is an $\varsigma_{1}$-vector given by

$$
F=\left[f_{0,0}, \ldots, f_{0, n_{2}}, \ldots, f_{n_{1}, 0}, \ldots, f_{n_{1}, n_{2}}\right]^{T},
$$

and $\Omega$ is defined in (2.2). The modified block-pulse coefficients, $f_{a_{1}, a_{2}}$, are obtained as

$$
f_{a_{1}, a_{2}}=\frac{1}{\ell\left(I_{a_{1}, \varepsilon}\right) \times \ell\left(I_{a_{2}, \varepsilon}\right)} \int_{I_{a_{1}, \varepsilon}} \int_{I_{a_{2}, \varepsilon}} f(x, y) d y d x
$$

where $\ell\left(I_{a_{1}, \varepsilon}\right)$ and $\ell\left(I_{a_{2}, \varepsilon}\right)$ are length of intervals $I_{a_{1}, \varepsilon}$ and $I_{a_{2}, \varepsilon}$ respectively. Similarly for every function $f(x, y, s, t)$, we can write

$$
f(x, y, s, t) \simeq \Omega(x, y)^{T} F_{\varepsilon} \Omega(s, t),
$$

where $F_{\varepsilon}$ is $2 \mathrm{D}-\mathrm{MBPF}$ coefficient matrix of dimension $\varsigma_{2}$.

\subsection{Ordinary perational matrix of 2D-MBPFs}

By the double integration of the vector $\Omega$ defined in $(2.2)$ we have $[8,10,11]$

$$
\int_{0}^{y} \int_{0}^{x} \Omega(s, t) d s d t \simeq P_{\varepsilon} \Omega(x, y)=\left[O_{\varepsilon,\left(n_{1}+1\right) \times\left(n_{1}+1\right)} \otimes O_{\varepsilon,\left(n_{2}+1\right) \times\left(n_{2}+1\right)}\right] \Omega(x, y),
$$

where $(x, y) \in D$ and $P_{\varepsilon}$ is $\varsigma_{2}$-ordinary operational matrix of integration for 2D-MBPFs so $O_{\varepsilon}$ is defined in [5]. In (2.5), $\otimes$ denotes the Kronecker product. By disjointness and orthogonality properties of 2D-MBPFs we have

$$
\int_{0}^{1} \int_{0}^{1} \Omega(s, t) \Omega^{T}(s, t) d s d t=\left(\begin{array}{ccccc}
\left(k_{1}-\varepsilon\right)\left(k_{2}-\varepsilon\right) & 0 & 0 & \ldots & 0 \\
0 & k_{1} k_{2} & 0 & \ldots & 0 \\
\vdots & \vdots & \ddots & \ddots & \vdots \\
0 & 0 & \ldots & k_{1} k_{2} & 0 \\
0 & 0 & \ldots & 0 & \varepsilon
\end{array}\right)=R_{\varepsilon}
$$

where $R_{\varepsilon}$ is the $\varsigma_{2}$-known matrix. 


\subsection{Stochastic operational matrix of 2D-MBPFs}

Similarly we obtain

$$
\int_{0}^{y} \int_{0}^{x} \Omega(s, t) d B(s) d B(t) \simeq P_{\varepsilon, s} \Omega(x, y)=\left[O_{\varepsilon, s,\left(n_{1}+1\right) \times\left(n_{1}+1\right)} \otimes O_{\varepsilon, s,\left(n_{2}+1\right) \times\left(n_{2}+1\right)}\right] \Omega(x, y),
$$

where $P_{\varepsilon, s}$ is the $\varsigma_{2}$-stochastic operational matrix of integration for 2D-MBPFs where $O_{\varepsilon, s}$ is defined in [3]. In the next sections, it is assumed that $T_{1}=T_{2}=1$.

\section{Method of solution}

Now, we solve (1.1) using 2D-MBPFs. By applying 2D-MBPFs approximates for functions

$$
f(x, y), V_{1}(x, y, s, t), V_{2}(x, y, s, t), V_{3}(x, y, s, t), g(x, y),
$$

we have

$$
\begin{gathered}
f=F_{\varepsilon}^{T} \Omega, \\
V_{1}=\Omega^{T}(x, y) \Gamma_{\varepsilon} \Omega(s, t), \\
V_{2}=\Omega^{T}(x, y) \Delta_{\varepsilon} \Omega(s, t), \\
V_{3}=\Omega^{T}(x, y) \Theta_{\varepsilon} \Omega(s, t),
\end{gathered}
$$

and

$$
g=G_{\varepsilon}^{T} \Omega,
$$

where the vectors $F_{\varepsilon}$ and $G_{\varepsilon}$ and matrices $\Gamma_{\varepsilon}, \Delta_{\varepsilon}$ and $\Theta_{\varepsilon}$ are the MBPFs coefficients of $f, g, V_{1}, V_{2}$ and $V_{3}$ respectively. In (3.1), $F_{\varepsilon}$ is $\varsigma_{1}$-known vector, also in (3.2), (3.3) and (3.4), $\Gamma_{\varepsilon}, \Delta_{\varepsilon}$ and $\Theta_{\varepsilon}$ are $\varsigma_{2}$-known matrices but in (3.5), $G_{\varepsilon}$ is $\varsigma_{1}$-unknown vector. In (1.1), To approximate Fredholm integral case from (3.2), (3.5) and using operational matrix $R_{\varepsilon}$ from (2.6) we get

$$
\begin{aligned}
\int_{0}^{1} \int_{0}^{1} V_{1} g d s d t & =\int_{0}^{1} \int_{0}^{1} \Omega^{T}(x, y) \Gamma_{\varepsilon} \Omega(s, t) \Omega^{T}(s, t) G_{\varepsilon} d s d t \\
& =\Omega^{T}(x, y) \Gamma_{\varepsilon}\left(\int_{0}^{1} \int_{0}^{1} \Omega(s, t) \Omega^{T}(s, t) d s d t\right) G_{\varepsilon} \\
& =\Omega^{T} \Gamma_{\varepsilon} R_{\varepsilon} G_{\varepsilon}=\left(\Gamma_{\varepsilon} R_{\varepsilon} G_{\varepsilon}\right)^{T} \Omega=U_{\varepsilon}^{T} \Omega,
\end{aligned}
$$

where $U_{\varepsilon}$ is an $\varsigma_{1}$-vector obtained from $\Gamma_{\varepsilon} R_{\varepsilon} G_{\varepsilon}$. Therefore for the approximation of the first 2D-integral we have

$$
\int_{0}^{1} \int_{0}^{1} V_{1} g d s d t \simeq U_{\varepsilon}^{T} \Omega
$$

In addition from (2.3), (3.3) and (3.5) we get [12]

$$
\begin{aligned}
\int_{0}^{y} \int_{0}^{x} V_{2} g d s d t \simeq \int_{0}^{y} \int_{0}^{x} \Omega^{T}(x, y) \Delta_{\varepsilon} \Omega(s, t) \Omega^{T}(s, t) G_{\varepsilon} d s d t & =\Omega^{T}(x, y) \Delta_{\varepsilon}\left(\int_{0}^{y} \int_{0}^{x} \Omega(s, t) \Omega^{T}(s, t) G_{\varepsilon} d s d t\right) \\
& =\Omega^{T} \Delta_{\varepsilon}\left(\int_{0}^{y} \int_{0}^{x} \tilde{G}_{\varepsilon} \Omega(s, t) d s d t\right)=\Omega^{T} \Delta_{\varepsilon} \tilde{G}_{\varepsilon}\left(\int_{0}^{y} \int_{0}^{x} \Omega(s, t) d s d t\right)
\end{aligned}
$$

where from (2.5) we arrive

$$
\int_{0}^{y} \int_{0}^{x} V_{2} g d s d t \simeq \Omega^{T} \Delta_{\varepsilon} \tilde{G}_{\varepsilon} P_{\varepsilon} \Omega
$$

in which $\Delta_{\varepsilon} \tilde{G}_{\varepsilon} P_{\varepsilon}$ is an $\varsigma_{2}$-matrix. From (2.4) we can write

$$
\int_{0}^{y} \int_{0}^{x} V_{2} g d s d t \simeq \hat{W}_{\varepsilon}^{T} \Omega
$$

where $\hat{W}_{\varepsilon}$ is an $\varsigma_{1}$-vector with components equal to the diagonal entries of matrix $\Delta_{\varepsilon} \tilde{G}_{\varepsilon} P_{\varepsilon}$. Similarly from (2.3), (3.4) and (3.5) we conclude

$$
\begin{aligned}
\int_{0}^{y} \int_{0}^{x} V_{3} g d B(s) d B(t) & \simeq \int_{0}^{y} \int_{0}^{x} \Omega^{T}(x, y) \Theta_{\varepsilon} \Omega(s, t) \Omega^{T}(s, t) G_{\varepsilon} d B(s) d B(t)=\Omega^{T}(x, y) \Theta_{\varepsilon}\left(\int_{0}^{y} \int_{0}^{x} \Omega(s, t) \Omega^{T}(s, t) G_{\varepsilon} d B(s) d B(t)\right) \\
& =\Omega^{T} \Theta_{\varepsilon}\left(\int_{0}^{y} \int_{0}^{x} \tilde{G}_{\varepsilon} \Omega(s, t) d B(s) d B(t)\right)=\Omega^{T} \Theta_{\varepsilon} \tilde{G}_{\varepsilon}\left(\int_{0}^{y} \int_{0}^{x} \Omega(s, t) d B(s) d B(t)\right),
\end{aligned}
$$

by using (2.7) we can arrive

$$
\int_{0}^{y} \int_{0}^{x} V_{3} g d B(s) d B(t) \simeq \Omega^{T} \Theta_{\varepsilon} \tilde{G}_{\varepsilon} P_{\varepsilon, s} \Omega
$$


in which $\Theta_{\varepsilon} \tilde{G}_{\varepsilon} P_{\varepsilon, s}$ is an $\varsigma_{2}$-matrix. From (2.4) we can write

$$
\int_{0}^{y} \int_{0}^{x} V_{3} g d B(s) d B(t) \simeq \hat{W}_{\varepsilon, s}^{T} \Omega
$$

where $\hat{W}_{\varepsilon, s}$ is an $\varsigma_{1}$-vector with components equal to the diagonal entries of matrix $\Theta_{\varepsilon} \tilde{G}_{\varepsilon} P_{\varepsilon, s}$. Applying (3.1), (3.5), (3.6), (3.7) and (3.8) in (1.1) give

$$
G_{\varepsilon}^{T} \Omega \simeq F_{\varepsilon}^{T} \Omega+\hat{U}_{\varepsilon}^{T} \Omega+\hat{W}_{\varepsilon}^{T} \Omega+\hat{W}_{\varepsilon, s}^{T} \Omega
$$

By replacing $\simeq$ with $=$, in (3.9) we can get

$$
G_{\varepsilon}-\hat{U}_{\varepsilon}-\hat{W}_{\varepsilon}-\hat{W}_{\varepsilon, s}=F_{\varepsilon}
$$

where after solving System (3.10), we can find $G_{\varepsilon}$ and get

$$
g=G_{\varepsilon}^{T} \Omega
$$

Then

$$
g \simeq g_{\varepsilon}=\frac{1}{\mu} \sum_{i=0}^{\mu-1} g_{\varepsilon_{i}}
$$

where $\varepsilon_{i}=\frac{i k}{\mu}, i=0(1)(\mu-1)$ is the estimation of the solution of (1.1) and $\mu$ is a positive integer.

\section{Error analysis}

In this section, we show that the convergence order of the proposed method is $\frac{1}{\mu n}$ by introducing several theorems. For convenience, we put $n_{1}=n_{2}=n$, so $k_{1}=k_{2}=\frac{1}{n}$.

Theorem 4.1. Suppose that $h$ is a differentiable function from $S \subset R^{2}$ into $R$, and for every $t \in S$

$$
\left\|h^{\prime}\right\|_{2} \leq \xi,
$$

where $\xi \in R$. Then

$$
|h(d)-h(c)| \leq \xi|d-c|,
$$

for all $c, d \in S$.

Proof. See [10].

Theorem 4.2. Assume that

$$
F_{n, \varepsilon}=\sum_{a=0}^{n} \sum_{b=0}^{n} \sum_{c=0}^{n} \sum_{d=0}^{n} F_{a, b, c, d} \omega_{a, b, c, d}
$$

and

$$
F_{a, b, c, d}=\frac{1}{\ell\left(I_{a, \varepsilon}\right) \ell\left(I_{b, \varepsilon}\right) \ell\left(I_{c, \varepsilon}\right) \ell\left(I_{d, \varepsilon}\right)} \times \int_{0}^{1} \int_{0}^{1} \int_{0}^{1} \int_{0}^{1} F \omega_{a, b, c, d} d t d s d y d x,
$$

where $a, b, c, d=0,1, \ldots, n$. Then the mean square error between $F$ and $F_{n, \varepsilon}$ on $(x, y, s, t) \in D_{a, b, c, d}$ reaches its minimum, moreover we have

$$
\int_{0}^{1} \int_{0}^{1} \int_{0}^{1} \int_{0}^{1} F^{2} d t d s d y d x=\sum_{a=0}^{\infty} \sum_{b=0}^{\infty} \sum_{c=0}^{\infty} \sum_{d=0}^{\infty} F_{a, b, c, d}^{2}\left\|\omega_{a, b, c, d}\right\|_{2}^{2}
$$

Proof. By using [11], we can easily prove this theorem.

Theorem 4.3. Assume $f$ is continuous and differentiable over district $[-k, 1+k] \times[-k, 1+k]$ and $f_{n, \varepsilon} ; \varepsilon_{i}=\frac{i k}{\mu}$ for $i=0,1, \ldots, \mu-1$ are correspondingly $2 D-M B P F s\left(\varepsilon_{0}\right)=2 D-B P F s, 2 D-M B P F s\left(\varepsilon_{1}\right), \ldots, 2 D-M B P F s\left(\varepsilon_{\mu-1}\right)$ expansions of $f$ based on $(n+1)^{2} 2 D-M B P F s$ over district $[0,1) \times[0,1)$ and $\bar{f}_{n, \mu}(x, y)=\frac{1}{\mu} \sum_{i=0}^{\mu-1} f_{n, \varepsilon_{i}}(x, y)$, then for sufficient large $n$ we have

$$
\left\|e_{\varepsilon}\right\|_{2} \leq \frac{\sqrt{2} N}{\mu n}
$$

therefore

$$
\left\|e_{\varepsilon}\right\|_{2}=O\left(\frac{1}{\mu n}\right),
$$

where $N$ is bounded of $\|D f\|_{2}$.

Proof. See [13]. 
Theorem 4.4. If $F$ be an enough smooth function on $S=[0,1)^{4}$ with $\|F\|_{2} \leq M$. Let

$$
\hat{F}_{n}=\hat{F}_{n, \varepsilon_{0}}=\sum_{a=1}^{n} \sum_{b=1}^{n} \sum_{c=1}^{n} \sum_{d=1}^{n} F_{a, b, c, d} \omega_{a, b, c, d},
$$

be $4 D-M B P F s\left(\varepsilon_{0}\right)=4 D-B P F$ s expansion of $F$ and

$$
e=F-\hat{F}_{n}
$$

then

$$
\left\|e_{\varepsilon}\right\|_{2}=O\left(\frac{1}{n}\right)
$$

Proof. We have

$$
e_{a, b, c, d}=F-F_{a, b, c, d} \phi_{a, b, c, d}=F-F_{a, b, c, d},
$$

where $\phi$ is the set of $4 \mathrm{D}-\mathrm{BPF}$ of dimension $n_{1} n_{2} n_{3} n_{4}$ and

$$
S_{a, b, c, d}=\left\{\frac{a-1}{n} \leq x<\frac{a}{n}, \frac{b-1}{n} \leq y<\frac{b}{n}, \frac{c-1}{n} \leq s<\frac{c}{n}, \frac{d-1}{n} \leq t<\frac{d}{n}\right\},
$$

and $(x, y, s, t) \in S_{a, b, c, d}$. By using the mean value theorem we get

$$
\begin{gathered}
\left\|e_{a, b, c, d}\right\|_{2}^{2}=\int_{(a-1) / n}^{a / n} \int_{(b-1) / n}^{b / n} \int_{(c-1) / n}^{c / n} \int_{(d-1) / n}^{d / n}\left(F-F_{a, b, c, d}\right)^{2} d t d s d y d x \\
=\frac{1}{n^{4}}\left(F\left(\gamma_{1}, \gamma_{2}, \gamma_{3}, \gamma_{4}\right)-F_{a, b, c, d}\right)^{2} ; \quad\left(\gamma_{1}, \gamma_{2}, \gamma_{3}, \gamma_{4}\right) \in S_{a, b, c, d} .
\end{gathered}
$$

We know

$$
F_{a, b, c, d}=\frac{1}{k^{4}} \int_{(a-1) k}^{a k} \int_{(b-1) k}^{b k} \int_{(c-1) k}^{c k} \int_{(d-1) k}^{d k} F_{a, b, c, d} d t d s d y d x
$$

therefore by using mean value theorem we have

$$
F_{a, b, c, d}=n^{4} \times \frac{1}{n^{4}} \times F\left(\theta_{1}, \theta_{2}, \theta_{3}, \theta_{4}\right) ; \quad\left(\theta_{1}, \theta_{2}, \theta_{3}, \theta_{4}\right) \in S_{a, b, c, d} .
$$

From Theorem 4.1 and involving (4.2) into (4.1) we obtain

$$
\left\|e_{a, b, c, d}\right\|_{2}^{2}=\frac{1}{n^{4}}\left(V\left(\gamma_{1}, \gamma_{2}, \gamma_{3}, \gamma_{4}\right)-V\left(\theta_{1}, \theta_{2}, \theta_{3}, \theta_{4}\right)\right)^{2} \leq \frac{1}{n^{4}} \times 4 k^{2} \times M^{2}=\frac{4 M^{2}}{n^{6}} .
$$

So

$$
\begin{aligned}
\|e\|_{2}^{2} & =\int_{0}^{1} \int_{0}^{1} \int_{0}^{1} \int_{0}^{1} e^{2} d t d s d y d x \\
& =\int_{0}^{1} \int_{0}^{1} \int_{0}^{1} \int_{0}^{1} \sum_{a=1}^{n} \sum_{b=1}^{n} \sum_{c=1}^{n} \sum_{d=1}^{n} e_{a b c d}^{2} d t d s d y d x+2 \sum_{a<a^{\prime}}^{n} \sum_{b<b^{\prime}}^{n} \sum_{c<c^{\prime}}^{n} \sum_{d<d^{\prime}}^{n} \int_{0}^{1} \int_{0}^{1} \int_{0}^{1} \int_{0}^{1} e_{a b c d} \times e_{a^{\prime} b^{\prime} c^{\prime} d^{\prime}} d t d s d y d x .
\end{aligned}
$$

Since for $a<a^{\prime}, b<b^{\prime}, c<c^{\prime}$ and $d<d^{\prime}$ we have

$$
S_{a, b, c, d} \cap S_{a^{\prime}, b^{\prime}, c^{\prime}, d^{\prime}}=\varnothing
$$

where (4.3) give

$$
\|e\|_{2}^{2}=\sum_{a=1}^{n} \sum_{b=1}^{n} \sum_{c=1}^{n} \sum_{d=1}^{n}\left\|e_{a b c d}\right\|_{2}^{2} \leq n^{4} \times \frac{4 M^{2}}{n^{6}}=\frac{1}{n^{2}} \times 4 M^{2},
$$

namely

$$
\|e\|_{2}=O\left(\frac{1}{n}\right)
$$

Theorem 4.5. Assume $F(x, y, s, t)$ is continuous and differentiable over district $[-k, 1+k] \times[-k, 1+k] \times[-k, 1+k] \times[-k, 1+k]$, moreover suppose $F_{n, \varepsilon_{i}}(x, y, s, t) ; \varepsilon_{i}=\frac{i k}{\mu}$ for $i=0,1, \ldots, \mu-1$ are correspondingly $4 D-M B P F s\left(\varepsilon_{0}\right)=4 D-B P F s, 4 D-M B P F s\left(\varepsilon_{1}\right), \ldots, 4 D-$ $\operatorname{MBPF}\left(\varepsilon_{\mu-1}\right)$ expansions of $F$ based on $(n+1)^{4} 4 D$-MBPFs over district $[0,1)^{4}$ and

$$
\bar{F}_{n, \mu}=\frac{1}{\mu} \sum_{i=0}^{\mu-1} F_{n, \varepsilon_{i}}
$$

then for sufficient large values $n$

$$
\left\|F-\bar{F}_{n, \mu}\right\|_{\infty} \lesssim \frac{1}{\mu} \max _{\varepsilon_{i}}\left\|F-\bar{F}_{n, \varepsilon_{i}}\right\|_{\infty}
$$


Proof. We consider partial differentials

$$
\frac{\partial F}{\partial x}, \frac{\partial F}{\partial y}, \frac{\partial F}{\partial s}, \frac{\partial F}{\partial t}
$$

in $D^{4}=\left[\frac{i-1}{n}, \frac{i+1}{n}\right)^{4}$ which are approximately equal to constants $A_{1}, A_{2}, A_{3}$ and $A_{4}$ respectively, where $n$ is so large. Also we use function, $z=A_{1} x+A_{2} y+A_{3} s+A_{4} t+B$ instead of $F$ in $D^{4}$. Now in the district $\left[\frac{i}{n}, \frac{i}{n}+\varepsilon_{1}\right)^{4}$ we have

$$
\begin{aligned}
\bar{F}_{n, \mu}(x, y, s, t) & =\frac{1}{\mu} \sum_{j=1}^{\mu-1} \frac{1}{16} \times\left[\left(A_{1}+A_{2}+A_{3}+A_{4}\right) \rho_{1}+B+\left(A_{1}+A_{2}+A_{3}\right) \rho_{1}+A_{4} \rho_{2}+B+\left(A_{1}+A_{2}+A_{4}\right) \rho_{1}+A_{3} \rho_{2}+B+\left(A_{1}+A_{2}\right) \rho_{1}\right. \\
& +\left(A_{3}+A_{4}\right) \rho_{2}+B+\left(A_{1}+A_{3}+A_{4}\right) \rho_{1}+A_{2} \rho_{2}+B+\left(A_{1}+A_{3}\right) \rho_{1}+\left(A_{2}+A_{4}\right) \rho_{2}+B+\left(A_{1}+A_{4}\right) \rho_{1}+\left(A_{2}+A_{3}\right) \rho_{2}+B+A_{1} \rho_{1} \\
& +\left(A_{2}+A_{3}+A_{4}\right) \rho_{2}+B+A_{1} \rho_{2}+\left(A_{2}+A_{3}+A_{4}\right) \rho_{1}+B+\left(A_{1}+A_{4}\right) \rho_{2}+\left(A_{2}+A_{3}\right) \rho_{1}+B+\left(A_{1}+A_{3}\right) \rho_{2}+\left(A_{2}+A_{4}\right) \rho_{1}+B \\
& +\left(A_{1}+A_{3}+A_{4}\right) \rho_{2}+A_{2} \rho_{1}+B+\left(A_{1}+A_{2}\right) \rho_{2}+\left(A_{3}+A_{4}\right) \rho_{1}+B+\left(A_{1}+A_{2}+A_{4}\right) \rho_{2}+A_{3} \rho_{1}+B \\
& \left.+\left(A_{1}+A_{2}+A_{3}\right) \rho_{2}+A_{4} \rho_{1}+B+\left(A_{1}+A_{2}+A_{3}+A_{4}\right) \rho_{2}+B\right] \\
& =\left(A_{1}+A_{2}+A_{3}+A_{4}\right)\left(\frac{\frac{i}{n}+\frac{i+1}{n}}{2}\right)+B-\frac{\left(A_{1}+A_{2}+A_{3}+A_{4}\right) k(\mu-1)}{2 \mu}
\end{aligned}
$$

where

$$
\rho_{1}=\left(\frac{i}{n}-\frac{j k}{\mu}\right)
$$

and

$$
\rho_{2}=\left(\frac{i+1}{n}-\frac{j k}{\mu}\right)
$$

Since $\frac{i+1}{n}=\frac{i}{n}+k$, we can reformulete (4.4) as

$$
\bar{F}_{n, \mu}=\left(A_{1}+A_{2}+A_{3}+A_{4}\right) \frac{i}{n}+B+\frac{\left(A_{1}+A_{2}+A_{3}+A_{4}\right) k}{2 \mu} .
$$

Also we have

$$
\max _{x, y, s, t \in\left[\frac{i}{n}, \frac{i}{n}+\varepsilon_{j}\right)}\left|F-\bar{F}_{n, \mu}\right| \simeq \max _{x, y, s, t \in\left[\frac{i}{n}, \frac{i}{n}+\varepsilon_{j}\right)}\left|A_{1} x+A_{2} y+A_{3} s+A_{4} t+B-\bar{F}_{n, \mu}\right|=\frac{\left(A_{1}+A_{2}+A_{3}+A_{4}\right) k}{2 \mu} .
$$

Therefore, we get

$$
\begin{gathered}
\max _{\varepsilon_{i}}\left\|F-F_{n, \varepsilon_{i}}\right\|_{\infty} \geq \underset{\varepsilon_{i}}{x, y, s, t \in D} \quad \max _{x, y, s, t \in D^{\prime}}\left|F-F_{n, \varepsilon_{i}}\right| \simeq \mid\left(A_{1}+A_{2}+A_{3}+A_{4}\right) \varpi_{1}+B-\frac{1}{16}\left[\left(A_{1}+A_{2}+A_{3}+A_{4}\right) \varpi_{1}+B\right. \\
+\left(A_{1}+A_{2}+A_{3}\right) \varpi_{1}+A_{4} \varpi_{2}+B+\left(A_{1}+A_{2}+A_{4}\right) \varpi_{1}+A_{3} \varpi_{2}+B+\left(A_{1}+A_{2}\right) \varpi_{1}+\left(A_{3}+A_{4}\right) \varpi_{2}+B+\left(A_{1}++A_{3}+A_{4}\right) \varpi_{1}+A_{2} \varpi_{2}+B \\
+\left(A_{1}+A_{3}\right) \varpi_{1}+\left(A_{2}+A_{4}\right) \varpi_{2}+B+\left(A_{1}+A_{4}\right) \varpi_{1}+\left(A_{2}+A_{3}\right) \varpi_{2}+B+A_{1} \varpi_{1}+\left(A_{2}+A_{3}+A_{4}\right) \varpi_{2}+B+A_{1} \varpi_{2}+\left(A_{2}+A_{3}+A_{4}\right) \varpi_{1}+B \\
+\left(A_{1}+A_{4}\right) \varpi_{2}+\left(A_{2}+A_{3}\right) \varpi_{1}+B+\left(A_{1}+A_{3}\right) \varpi_{2}+\left(A_{2}+A_{4}\right) \varpi_{1}+B+\left(A_{1}+A_{3}+A_{4}\right) \varpi_{2}+A_{2} \varpi_{1}+B+\left(A_{1}+A_{2}\right) \varpi_{2}+\left(A_{3}+A_{4}\right) \varpi_{1}+B \\
\left.+\left(A_{1}+A_{2}+A_{4}\right) \varpi_{2}+A_{3} \varpi_{1}+B+\left(A_{1}+A_{2}+A_{3}\right) \varpi_{2}+A_{4} \varpi_{1}+B+\left(A_{1}+A_{2}+A_{3}+A_{4}\right) \varpi_{2}+B\right] \mid \\
=\frac{\left(A_{1}+A_{2}+A_{3}+A_{4}\right)}{2} k,
\end{gathered}
$$

where $\varpi_{1}=\frac{i}{n}, \frac{i}{n}+k=\varpi_{2}, D=\left[\frac{i-1}{n}, \frac{i+1}{n}\right)$ and $D^{\prime}=\left[\frac{i}{n}, \frac{i}{n}+k\right)$. From (4.5) and (4.6) we get

$$
\left\|F-\bar{F}_{n, \mu}\right\|_{\infty} \lesssim \frac{1}{\mu} \max _{\varepsilon_{i}}\left\|F-\bar{F}_{n, \varepsilon_{i}}\right\|_{\infty} .
$$

\section{Remark 4.6. Let}

$$
e_{n, \varepsilon}=F-\bar{F}_{n, \mu}
$$

and

$$
e_{n}=F-\bar{F}_{n},
$$

then from Theorem 4.2, Theorem 4.4 and Theorem 4.5 we have

$$
\left\|e_{n, \varepsilon}\right\|_{2} \leqslant \frac{2 M}{\mu n}
$$

also we can write

$$
\lim _{n \rightarrow+\infty} F_{n, \varepsilon_{i}}=F
$$


Theorem 4.7. If $g$ be the exact solution of (1.1) and $\hat{g}_{n, \mu}(x, y)$ be the $2 D$-MBPFs approximate solution of it. Also

(1) $\|g\|_{2} \leq \alpha, \quad(s, t) \in[0,1)^{2}$,

(2) $\left\|V_{i}\right\|_{2} \leq \beta_{i}, i=1,2,3,(x, y, s, t) \in[0,1)^{4}$,

(3) $W_{1}(x, y)=\sup _{x \in[0,1)} x \times \sup y$,

(4) $W_{2}(x, y)=\sup _{x \in[0,1)}|B(x)| \times \sup _{y \in[0,1)}|B(y)|$,

(5) $\left[\beta_{1}+\beta_{2}+\frac{2 \beta_{1}+2 \beta_{2}}{\mu n}+\left(\beta_{3}+\frac{2 \beta_{3}}{\mu n}\right) \times W_{2}(x, y)\right]<1$,

then

$$
\left\|g-\hat{g}_{n}\right\|_{2}=O\left(\frac{1}{\mu n}\right)
$$

Proof. From (1.1), we get

$$
\begin{gathered}
g-\hat{g}_{n, \mu}=f-\hat{f}_{n, \mu}+\int_{0}^{1} \int_{0}^{1}\left(V_{1} g-\hat{V}_{1, n, \mu} \hat{g}_{n, \mu}\right) d s d t+\int_{0}^{y} \int_{0}^{x}\left(V_{2} g-\hat{V}_{2, n, \mu} \hat{g}_{n, \mu}\right) d s d t \\
+\int_{0}^{y} \int_{0}^{x}\left(V_{3}(x, y, s, t) g(s, t)-\hat{V}_{3, n, \mu} \hat{g}_{n, \mu}\right) d B(s) d B(t),
\end{gathered}
$$

so the mean value theorem give

$$
\left\|g-\hat{g}_{n, \mu}\right\|_{2} \leq\left\|f-\hat{f}_{n, \mu}\right\|_{2}+\left\|V_{1} g-\hat{V}_{1, n, \mu} \hat{g}_{n, \mu}\right\|_{2}+x y\left\|V_{2} g-\hat{V}_{2, n, \mu} \hat{g}_{n, \mu}\right\|_{2}+B(x) B(y)\left\|V_{3} g-\hat{V}_{3, n, \mu} \hat{g}_{n, \mu}\right\|_{2} .
$$

By using Remark 4.6 and two first hypothesises, we obtain

$$
\begin{aligned}
\left\|V_{1} g-\hat{V}_{1, n, \mu} \hat{g}_{n, \mu}\right\|_{2} & \leq\left\|V_{1}\right\|_{2}\left\|g-\hat{g}_{n, \mu}\right\|_{2}+\left\|V_{1}-\hat{V}_{1, n, \mu}\right\|_{2}\left(\left\|g-\hat{g}_{n, \mu}\right\|_{2}+\|g\|_{2}\right) \\
& \leq \beta_{1}\left\|g-\hat{g}_{n, \mu}\right\|_{2}+\frac{2 \beta_{1}}{\mu n}\left(\left\|g-\hat{g}_{n, \mu}\right\|_{2}+\alpha\right)=\left(\beta_{1}+\frac{2 \beta_{1}}{\mu n}\right)\left\|g-\hat{g}_{\mu n}\right\|_{2}+\frac{2 \beta_{1}}{\mu n} \alpha .
\end{aligned}
$$

Similarly we have

$$
\left\|V_{2} g-\hat{V}_{2, n, \mu} \hat{g}_{n, \mu}\right\|_{2}=\left(\beta_{2}+\frac{2 \beta_{2}}{\mu n}\right)\left\|g-\hat{g}_{n, \mu}\right\|_{2}+\frac{2 \beta_{2}}{\mu n} \alpha
$$

and

$$
\left\|V_{3} g-\hat{V}_{3, n, \mu} \hat{g}_{n, \mu}\right\|_{2}=\left(\beta_{3}+\frac{2 \beta_{3}}{\mu n}\right)\left\|g-\hat{g}_{n, \mu}\right\|_{2}+\frac{2 \beta_{3}}{\mu n} \alpha .
$$

Substituting (4.8), (4.9) and (4.10) in (4.7) and Theorem 4.3 conclude

$$
\begin{gathered}
\left\|g-\hat{g}_{n, \mu}\right\|_{2} \leq \frac{\sqrt{2} N}{\mu n}+\left[\left(\beta_{1}+\frac{2 \beta_{1}}{\mu n}\right)\right. \\
\left.\left\|g-\hat{g}_{n, \mu}\right\|_{2}+\frac{2 \beta_{1}}{\mu n} \alpha\right]+x y\left[\left(\beta_{2}+\frac{2 \beta_{2}}{\mu n}\right)\left\|g-\hat{g}_{n, \mu}\right\|_{2}+\frac{2 \beta_{2}}{\mu n} \alpha\right] \\
+B(x) B(y)\left[\left(\beta_{3}+\frac{2 \beta_{3}}{\mu n}\right)\left\|g-\hat{g}_{n, \mu}\right\|_{2}+\frac{2 \beta_{3}}{\mu n} \alpha\right] .
\end{gathered}
$$

By taking sup and Hypothesises 3 and 4, we have

$$
\begin{aligned}
\left\|g-\hat{g}_{n, \mu}\right\|_{2} \leq \frac{\sqrt{2} N}{\mu n}+\left[\left(\beta_{1}+\frac{2 \beta_{1}}{\mu n}\right) \sup _{s \leq x, t \leq y}\right. & \left.\left\|g-\hat{g}_{n, \mu}\right\|_{2}+\frac{2 \beta_{1}}{\mu n} \alpha\right]+W_{1}(x, y)\left[\left(\beta_{2}+\frac{2 \beta_{2}}{\mu n}\right) \sup _{s \leq x, t \leq y}\left\|g-\hat{g}_{n, \mu}\right\|_{2}+\frac{2 \beta_{2}}{\mu n} \alpha\right] \\
& +W_{2}(x, y)\left[\left(\beta_{3}+\frac{2 \beta_{3}}{\mu n}\right) \sup _{s \leq x, t \leq y}\left\|g-\hat{g}_{n, \mu}\right\|+\frac{2 \beta_{3}}{\mu n} \alpha\right],
\end{aligned}
$$

so

$$
\left\|g-\hat{g}_{n, \mu}\right\|_{2} \leq \frac{\frac{\sqrt{2} N+2 \beta_{1} \alpha+2 \beta_{2} \alpha}{\mu n}+\frac{2 \beta_{3} \alpha}{\mu n} \times W_{2}(x, y)}{1-\left[\beta_{1}+\beta_{2}+\frac{2 \beta_{1}+2 \beta_{2}}{\mu n}+\left(\beta_{3}+\frac{2 \beta_{3}}{\mu n}\right) \times W_{2}(x, y)\right]},
$$

and from the boundedness of Brownian motion we get

$$
\left\|g-\hat{g}_{n, \mu}\right\|_{2}=O\left(\frac{1}{\mu n}\right) .
$$




\begin{tabular}{|c|c|c|c|c|}
\hline$n$ & $\mu$ & $\bar{g}$ & $\bar{e}$ & $(L, U)$ \\
\hline 2 & 1 (BPFs) & 0.488705 & 0.188705 & $(0.487912,0.489498)$ \\
& 3 (MBPFs) & 0.397704 & 0.097704 & $(0.390937,0.404471)$ \\
\hline 3 & 1 (BPFs) & 0.327094 & 0.027093 & $(0.326647,0.327540)$ \\
& 3 (MBPFs) & 0.316383 & 0.016382 & $(0.304258,0.328508)$ \\
\hline 4 & 1 (BPFs) & 0.246046 & 0.053945 & $(0.245386,0.246705)$ \\
& 3 (MBPFs) & 0.582630 & 0.034799 & $(0.222132,0.343128)$ \\
\hline 5 & 1 (BPFs) & 0.400235 & 0.100235 & $(0.399732,0.400738)$ \\
& 3 (MBPFs) & 0.360730 & 0.060730 & $(0.356168,0.365292)$ \\
\hline
\end{tabular}

Table 1: Results in $(0.1,0.2)$

\begin{tabular}{|c|c|c|c|c|}
\hline$n$ & $\mu$ & $\bar{g}$ & $\bar{e}$ & $(L, U)$ \\
\hline 2 & 1 (BPFs) & 0.996151 & 0.296151 & $(0.987828,1.004470)$ \\
& 3 (MBPFs) & 0.906776 & 0.206776 & $(0.870497,0.943055)$ \\
\hline 3 & 1 (BPFs) & 0.997234 & 0.297234 & $(0.976630,1.017840)$ \\
& 3 (MBPFs) & 0.880689 & 0.180689 & $(0.833557,0.927822)$ \\
\hline 4 & 1 (BPFs) & 0.792689 & 0.092689 & $(0.788890,0.796488)$ \\
& 3 (MBPFs) & 0.751917 & 0.051917 & $(0.733360,0.770475)$ \\
\hline 5 & 1 (BPFs) & 0.799899 & 0.099899 & $(0.799426,0.800373)$ \\
& 3 (MBPFs) & 0.782178 & 0.082178 & $(0.776386,0.787971)$ \\
\hline
\end{tabular}

Table 2: Results in $(0,0.7)$

\section{Numerical example}

We consider a numerical example to illustrate the efficiency of the MBPFs method. Consider the 2D-linear stochastic Volterra-Fredholm integral equation

$$
g(x, y)=f(x, y)+\int_{0}^{1} \int_{0}^{1}(x y s t) g(s, t) d s d t+\int_{0}^{y} \int_{0}^{x}(x y s t) g(s, t) d s d t+\int_{0}^{y} \int_{0}^{x}(x y s t) g(s, t) d B(s) d B(t),
$$

where

$$
f(x, y)=x+y-\frac{x y}{3}-\frac{x^{3} y^{3}(x+y)}{6}-\left(x^{3} y^{2}+x^{2} y^{3}\right) B(x) B(y)+B(x)\left(x^{3} y+2 x^{2} y\right) \int_{0}^{y} B(t) d t+B(y)\left(y^{3} x+2 y^{2} x\right) \int_{0}^{x} B(s) d s
$$

with the exact solution

$$
g(x, y)=x+y .
$$

The solution mean $(\bar{g}(x, y))$, error mean $(\bar{e}(x, y))$ and $\% 95$ confidence interval $(L, U)$ at arbitrary points $(0.1,0.2)$ and $(0,0.7)$ for some values of $n$ and $\mu$ are shown in Table 1 and Table 2 . In this tables by the comparison between the computed results by the presented method and the BPFs method we will see that in the MBPFs method we achieve the good accuracy by increasing $\mu$. You can see three-dimensional graphs of this example in Fig. 5.1 and Fig. 5.2.

\section{Conclusion}

In this paper, we have successfully developed the 2D-MBPFs numerical method for approximate a solution for 2D-linear stochastic Volterra-Fredholm integral equations. The numerical results represent that $\bar{e}$ in new method is lesser from $\bar{e}$ in $\mathrm{BPF}$ method.

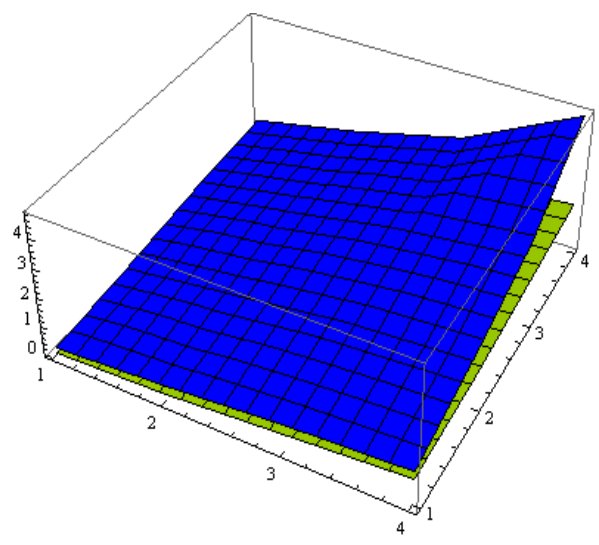

Figure 5.1: $(n=3)$ 


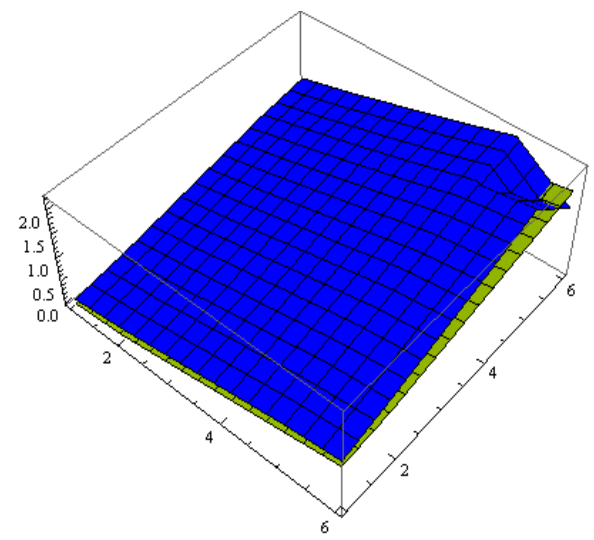

Figure 5.2: $(n=5)$

\section{References}

[1] K. E. Atkinson, The Numerical Solution of Integral Equations of the Second Kind, Cambridge University Press, 1997.

[2] A. J. Jerri, Introduction to Integral Equations with Applications, John Wiley and Sons, INC, 1999.

[3] M. Fallahpour, M. Khodabin, K. Maleknejad, Approximation solution of two-dimensional linear stochastic Volterra-Fredholm integral equation via two-dimensional Block-pulse functions, Int. J. Indus. Math., 8(4) (2016), IJIM-00774.

[4] M. Fallahpour, M. Khodabin, K. Maleknejad, Approximation solution of two-dimensional linear stochastic fredholm integral equation by applying the Haar wavelet, Math. Model. Comp., 5 (2015), 361- 372.

[5] M. Fallahpour, M. Khodabin, K. Maleknejad, Theoretical error analysis and validation in numerical solution of two-dimensional linear stochastic Volterra-Fredholm integral equation by applying the block-pulse functions, Cog. Math., 4 (2017), 1296750.

[6] M. Fallahpour, M. Khodabin, R. Ezzati, A new computational method based on Bernstein operational matrices for solving two-dimensional Linear stochastic Volterra integral equations, Differ. Equat. Dynam. Syst., (2019), doi.org/10.1007/s12591-019-00474-y.

[7] M. Fallahpour, M. Khodabin, K. Maleknejad, Theoretical error analysis of solution for two-dimensional stochastic Volterra integral equations by Haar wavelet, Int. J. Appl. Comput. Math, (2019), doi.org/10.1007/s40819-019-0739-3.

[8] F. Mirzaee, E. Hadadiyan, Using modified two-dimensional block-pulse functions for the numerical solution of nonlinear two-dimensional Volterra integral equations, J. Hyperst., 3(1) (2014), 68-80.

[9] K. Maleknejad, B. Rahimi, Modification of block pulse functions and their application to solve numerically Volterra integral equation of the first kind, Com. Non. Sci. Num. Sim, 16 (2011), 2469-2477.

[10] K. Maleknejad, S. Sohrabi, B. Baranji, Two-dimensional PCBFs: Application to nonlinear Volterra integral equations, Proce. Wor. Cong. Engin., (2009), Vol II WCE 2009, July 1 - 3.

[11] Z. H. Jiang, W. Schaufelberger, Block Pulse Functions and Their Applications in Control Systems, Springer-Verlag, 1992.

[12] K. Maleknejad, M. Khodabin, F. Hosseini Shekarabi, Modified block pulse functions for numerical solution of stochastic Volterra integral equations, Appl. Math., (2014), doi. org/10.1155/2014/469308.

[13] F. Mirzaee, E. Hadadiyan, Approximate solutions for mixed nonlinear Volterra-Fredholm type integral equations via modified block-pulse functions, J. Assoc. Arab. Uni. Bas. Appl. Sci., 12 (2012), 65-73. 